\title{
BPS domain walls from backreacted orientifolds
}

\author{
Johan Blåbäck, ${ }^{a}$ Bert Janssen, ${ }^{b}$ Thomas Van Riet $^{c}$ and Bert Vercnocke ${ }^{d}$ \\ ${ }^{a}$ Institutionen för fysik och astronomi, Uppsala Universitet, \\ Box 516, SE-751 08 Uppsala, Sweden \\ ${ }^{b}$ Departamento de Física Teórica y del Cosmos \\ and Centro Andaluz de Física de Partículas Elementales, Universidad de Granada, \\ 18071 Granada, Spain \\ ${ }^{c}$ Instituut voor Theoretische Fysica, Katholieke Universiteit Leuven, \\ Celestijnenlaan 200D B-3001 Leuven, Belgium \\ ${ }^{d}$ Institute of Theoretical Physics, University of Amsterdam, \\ Science Park, Postbus 94485, 1090 GL Amsterdam, Netherlands \\ E-mail: johan.blaback@physics.uu.se, bjanssen@ugr.es, \\ thomasvr@itf.fys.kuleuven.be, bert.vercnocke@uva.nl
}

Abstract: Compactifications with D-brane and orientifold sources lead to standard gauged supergravity theories if the sources are smeared over the internal directions. It is therefore of interest to find how the solutions described by the gauged supergravity are altered by properly localising the sources. In this paper we analyse this for BPS domain wall solutions in the seven-dimensional gauged supergravity obtained from an O6 toroidal orientifold in massive IIA supergravity. This is one of the simplest no-scale supergravities that can be constructed and analysed in full detail. We find the BPS domain walls when the O6 planes are smeared. When the O6 planes are localised the domain wall solutions live in a warped compactification and we present the first-order equations these domain walls obey in 10 dimensions. In order to get explicit expressions we also consider the noncompact versions of the solutions for which the O6 planes have been traded for D6 branes and we recover the gauged supergravity expressions for the domain walls in the leading terms of the warp factor. Through T-duality we obtain partially localised solutions for compactifications to four dimensions using O3 planes with 3-form fluxes.

KEYwords: Flux compactifications, D-branes, Superstring Vacua

ARXIV EPRINT: 1312.6125 


\section{Contents}

1 Introduction 1

$2 \mathrm{D} p / \mathrm{O} p$ sources smeared and localised $\quad 4$

3 Domain walls in the smeared limit $\quad 6$

$\begin{array}{ll}3.1 \text { Seven-dimensional gauged supergravity } & 7\end{array}$

3.2 BPS and fake BPS domain walls 8

4 Full backreaction in ten-dimensional supergravity $\quad 9$

$\begin{array}{llr}4.1 & 1 / 4 \text { BPS solutions in massive IIA supergravity } & 10\end{array}$

$\begin{array}{lll}4.2 & \text { Smeared limit in } 10 \text { dimensions } & 11\end{array}$

$\begin{array}{ll}4.3 & \text { Warped domain walls } \\ & 4.3 .12\end{array}$

$\begin{array}{ll}\text { 4.3.1 Warping for a simple class of domain walls } & 12\end{array}$

4.3.2 Warping for all supersymmetric domain walls 13

5 A 4D example from O3 planes $\quad 14$

$\begin{array}{lll}5.1 \text { Smeared O3 compactification } & 15\end{array}$

$\begin{array}{lll}5.2 & \text { BPS domain walls from smeared O3 planes } & 15\end{array}$

$\begin{array}{lll}5.3 & \text { Warped solutions from localised O3 planes } & 16\end{array}$

$\begin{array}{llr}6 & \text { Discussion } & 16\end{array}$

$\begin{array}{ll}\text { A } \mathcal{N}=1 \text { supergravity formulation } & 17\end{array}$

\section{Introduction}

Flux compactifications of string theory that are of phenomenological interest typically involve the use of space-filling D-brane or orientifold sources. One of the most relevant reasons is that such sources seem necessary for flux solutions with a separation between the Kaluza-Klein (KK) scale and the scale of the vacuum energy [1,2]. If such a separation is absent, the vacuum does not appear as four-dimensional to an observer that can do experiments with energy at least as large as the vacuum energy. A second reason for the use of sources is their importance in de Sitter model building [3-5]. For both the issue of scale separation and dS model building the sources should have net negative tension (orientifold planes), at least at leading order in the $\alpha^{\prime}$ and $g_{s}$ expansion $[1,6,7]$. A third reason is their importance in creating sufficient warping, which in turn is useful in attempts to solve the hierarchy problem [8], or to warp down supersymmetry-breaking terms [9]. Other reasons are that D-branes are used in type II constructions of standard model building $[10,11]$ and they are a source of supersymmetry breaking. 
When these sources are parallel, or when they allow an F-theory understanding, the backreaction can be computed exactly, at least for supersymmetric vacua $[8,12,13]$. Unfortunately some of the most intriguing flux solutions necessarily involve intersecting sources whose backreaction has not yet been computed. Most notable are the flux compactifications of type IIA [14-17] and type IIB supergravity [1, 18, 19] to supersymmetric $A d S_{4}$ vacua which have astonishing features: they allow moduli stabilisation at the classical level, an arbitrary separation of scales together with arbitrary weak coupling, small cosmological constant and large volume such that these solutions are under maximal control. Unfortunately these solutions have only been studied in the smeared limit (see [20] for a discussion on that matter) and little progress has been made on how the backreaction of the localised sources could be described, apart from the investigation in [21]. It has even been suggested that the backreaction can invalidate the existence of the solutions [22] or be very large such that the smeared limit is far from the actual solution [23]. This clearly indicates that computing the backreaction of space-filling sources in flux solutions is of crucial importance.

There are two approaches to this problem: one can simply attempt to compute the exact backreaction. Or one could try to understand the effect of the backreaction in the low energy effective action. This last option implies one does not need to know the full backreaction, but rather needs to understand its integrated effect. These attempts go by the name of warped effective field theory (WEFT) and a sample of papers on this topic is [24-27]. However, several important issues that depend on local features, like those raised in [21-23] for supersymmetric AdS vacua or the issues related to the stability of the anti-branes [28] in the KKLT scenario [9], cannot be addressed by the integrated approach of WEFT. ${ }^{1}$ Therefore we are interested in computing the exact backreaction.

There are at least two relevant circumstances for which exact backreaction has not been computed: dynamical solutions in models with parallel sources and both vacuum and dynamical solutions in models with intersecting sources. In this paper we address the first of these. We compute the backreaction of orientifold planes in BPS domain wall solutions of no-scale supergravity theories. Apart from the classical no-scale Minkowski vacuum one can consider solutions that involve non-constant fields. Domain walls are such a particular class of solutions that probe the structure of the scalar potential, since the scalar fields of such solutions are non-constant in space. We found it natural to treat the BPS domain walls first, due to the supersymmetry conditions. One of our motivations to study domain walls, apart from the technical reasons mentioned above, is their interpretation as tunnelling processes in flux vacua landscapes [29]. It has been argued that the warping can be relevant in the tunnelling process [30], which was ignored in the gauged supergravity analysis of [29]. The arguments of [30] rely on WEFT, but it would clearly be beneficial to have the full 10-dimensional solution describing the tunnelling process. In this paper we will not verify the effect of warping on the tunnelling process but instead provide the technical tools necessary for this.

\footnotetext{
${ }^{1}$ The stability of these branes is questioned as a consequence of the singular three-form fluxes when the anti-branes backreact [31-35]. A review on the state of the art of backreaction computations can be found in $[36]$.
} 
This paper is a continuation of earlier work [37], in which we formulated a simple supergravity model and were able to compute the backreaction for a rather simple domain wall flow. This model is the simplest no-scale supergravity we are aware of and it lives in seven space-time dimensions. It can be found from a compactification of massive IIA supergravity on $\mathbb{T}^{3} / \mathbb{Z}_{2}$, with 8 space-filling $\mathrm{O} 6$ planes and the appropriate $H_{3}$ flux and Romans mass contribution $F_{0}$ to cancel the RR tadpole. The no-scale Minkowski vacuum breaks supersymmetry, in a way similar to the no-scale solutions in four dimensions from IIB orientifolds with ISD fluxes that contain a $(0,3)$-piece [8] (in fact our model is Tdual to such a set-up). The simplest domain wall flows through moduli space ${ }^{2}$ and its backreaction was computed exactly [37]. In fact, it already existed in an earlier paper [38], but was left without interpretation at the time. Since the flow occurs in the moduli space, such a domain wall does not probe the potential energy. In this paper we consider all BPS domain wall flows, hence also those that move upward in the potential and compute their backreaction. Again we can rely on earlier investigations of BPS solutions in massive IIA [39] that we give a new interpretation.

The practical motivation for studying the O6 massive IIA compactification to seven dimensions is its simplicity, allowing us to classify all BPS domain walls and find their explicit warped versions. However, this simple flux compactification is interesting in its own right, since it relates to the debate on the consistency of $\mathrm{O} 6$ planes in massive IIA string theory [22]. There is no known lift of the $\mathrm{O} 6$ planes in massive IIA to 11 dimensions where the singularity of the orientifold space time could be resolved. Recently, in [21] an intriguing observation was made that $\mathrm{O} 6$ solutions in massive IIA could be regular already prior to any possible uplift. Our massive IIA Minkowski solution is simple enough to verify this idea explicitly within a compact setting.

The rest of this paper is organised as follows. We start with section 2, where we describe a formal map between compact $(\mathrm{O} p)$ and non-compact $(\mathrm{D} p)$ solutions since the non-compact ones allow a more analytic treatment of backreaction. In section 3 we compactify massive IIA with smeared O6 planes to seven-dimensional gauged supergravity and we solve for the BPS domain walls. We compute the backreaction of the localised sources in these domain wall solutions in section 4 . To that end we review all the non-compact $1 / 4$ BPS solutions in massive IIA that possess the proper symmetries to be interpreted as domain walls and we give the corresponding compact orientifold solutions. Only a subset of these solutions describe the backreaction of the smeared domain walls. Unfortunately explicit solutions are not possible, not even for the vacuum solution, and we present instead the Ansatz and the first-order equations for the solutions in 10-dimensions. As usual not all equations are first-order and the equation that determines the warp factor remains second-order. When we flip the sign of the charge and tension we find explicit non-compact D6 solutions that have the domain wall solutions of the gauged supergravity on their worldvolume. The domain walls can be recognized by the leading terms in the warp factor of the solution.

\footnotetext{
${ }^{2}$ In this paper moduli space refers to the space of continuous massless deformations of the solution.
} 
In section 5 we initiate the same program for four-dimensional IIB orientifold compactifications, which are of more interest. By a simple T-duality we obtain the solutions corresponding to the partially backreacted four-dimensional domain walls. Section 6 contains a discussion and various avenues for new research.

\section{$2 \quad \mathrm{D} p / \mathrm{O} p$ sources smeared and localised}

Solutions describing orientifold compactifications tend to be (partly) implicit because the equations of motion involve the Laplacian on the internal space. Solutions to these generalised Laplace equations are not analytically known in general. When certain problems require explicit expressions it becomes desirable to find a way around this. A common technique is to describe the solution only locally and not to worry about compactness. For example one could consider polar coordinates on a torus and make an Ansatz that only depends on the radial variable. The generalised Laplace equation can then often be solved analytically and almost always numerically. This solution will however not be complete since polar coordinates do not cover the whole torus. ${ }^{3}$ For a class of models, including the one we discuss in this paper, there is a different technique to find analytic expressions. This relies on changing the $\mathrm{O} p$ planes with $\mathrm{D} p$ branes as we now explain.

Consider the following Bianchi identity:

$$
\mathrm{d} F_{8-p}=H_{3} \wedge F_{6-p}+\rho_{p}
$$

where the charge distribution $\rho_{p}$ is given by $\rho_{p}=Q_{p} \delta_{p}$ for a localised $\mathrm{O} p / \mathrm{D} p$ source. If the space $M$ transverse to the $\mathrm{O} p / \mathrm{D} p$ source is compact then we can derive the following equation by integration

$$
Q_{p}=-\int_{M} H_{3} \wedge F_{6-p} .
$$

Hence, if no fluxes $H_{3}$ and $F_{6-p}$ are present we get the usual consequence of Gauss' law that a compact space cannot be charged. In the presence of fluxes this can be remedied by adding the exact opposite charge dissolved in fluxes. If, on the other hand, the space is non-compact, there is no such constraint. Almost all of the BPS flux solutions in the literature are such that for $\mathrm{D} p$ branes and $\mathrm{O} p$ planes the following relation between the signs exists

$$
\begin{aligned}
& \operatorname{sign}\left[Q_{O p}\right]=-\operatorname{sign}\left[\int_{M} H_{3} \wedge F_{6-p}\right], \\
& \operatorname{sign}\left[Q_{D p}\right]=+\operatorname{sign}\left[\int_{M} H_{3} \wedge F_{6-p}\right] .
\end{aligned}
$$

Therefore a generic BPS flux solution is compact when $\mathrm{O} p$ planes are invoked and noncompact for $\mathrm{D} p$ branes. In what follows we refer to such models as 'vanilla models'. A simple intuition for (2.3), (2.4) was presented in [12]. One can think of the BPS orientifold solutions as solutions that have a balance in forces between two objects: the localised

\footnotetext{
${ }^{3}$ This is well known for the non-compact Klebanov-Strassler conifold solution [40], which should be a local description of a highly warped conifold point in a compact CY orientifold construction of [8].
} 
(delta-peaked) orientifold sources and the smooth flux distribution $H \wedge F_{6-p}$ which carries opposite charge and tension. Since the tensions of sources and fluxes are opposite the gravitational repulsion between these objects is exactly counterbalanced by the electromagnetic attraction due to the opposite charges. When the $\mathrm{O} p$ planes are replaced with $\mathrm{D} p$-branes, which have opposite charge and tension, the same balance holds, but now gravity is attractive and electromagnetism repulsive. Because of this no-force condition the vanilla models allow a formal map between compact and non-compact solutions. For a given orientifold setup one can simply change the $\mathrm{O} p$ charge (and tension) distribution by a $\mathrm{D} p$ distribution.

$$
O p \leftrightarrow D p
$$

This interchange has the opposite sign of charge and tension and hence a compact solution will not be possible anymore if the $\mathrm{O} p$ setup was compact as a consequence of Gauss' law (2.2) and (2.4). The formal map (2.5) does not affect the bulk equations of motion, only the boundary conditions (the delta sources one has to connect to). Since compactness is lost one can hope to have some radial variable to make the generalised Laplace equations into ODE's. Perhaps the simplest example of such a correspondence is the no-scale sevendimensional Minkowski vacuum in massive IIA supergravity with space-filling O6 planes first described in [12]. Its non-compact version coincides with an earlier supergravity solution that describes a D6 brane in massive IIA supergravity, see [38] and [39].

The main result of this paper is that the non-compact $\mathrm{D} p$ solutions indeed carry the information of orientifold compactifications and their associated gauged supergravity description. The domain wall flows of the gauged supergravity that follows from a smeared orientifold compactification give rise to profiles for certain fields in ten dimensions, denoted $\Phi_{10}$, that depend on the domain wall coordinate $z$

$$
\Phi_{10 d}=\phi_{7 d}(z)
$$

where the expression $\phi_{7 d}(z)$ is a certain combination of fields of the seven-dimensional supergravity. When we then apply the formal map (2.5) and construct the localised $\mathrm{D} p$ solution we find an expression of the form:

$$
\Phi_{10 d}(r, z)=\sum_{i=-N}^{\infty} a_{i}(z) r^{i}, \quad \text { where } a_{0}(z)=\phi_{7 d}(z) .
$$

The general solution is described by a Laurent expansion in the radial coordinate $r$ of the three-dimensional transverse space and the $r$-independent term coincides exactly with the expression obtained from the seven-dimensional gauged supergravity. The coefficients in the Laurent expansion obey certain iterative relations and when combined with the requirement that the solution smears properly, all of the coefficients are defined uniquely. For compact $\mathrm{O} p$ solutions the situation is very similar. The main difference is that the Laurent expansion is traded for a Fourier expansion on the internal torus and again the constant piece coincides with the gauged supergravity. This result was to be expected since the fields in the gauged supergravity typically correspond to the lowest Fourier mode, which is constant on the compact space. Unfortunately we have not been able to find 
a simple iterative set of differential equations for the Fourier coefficients. This makes the non-compact solution more attractive since the Laurent coefficients can be computed very easily. We expect that (2.7) holds for all vanilla models. It is furthermore expected that the genuine compact solution can be described in the vicinity of the O6 plane by a rotationally symmetric solution. Then one can write down the solution in terms of the local radial coordinate on the 3 -torus. Up to the sign of the $1 / r$ term this coincides with the non-compact D6 solutions. This offers a different way to justify the formal map (2.5).

The rest of the paper is devoted to the explicit derivation of this result for the O6 compactification to $D=7$.

Before we continue we briefly want to discuss what happens to non-vanilla models. In [12] a recipe for AdS solutions was given that relies on solving the RR tadpole (2.2) using D-brane sources instead of orientifold planes. Such solutions have a complicated backreaction [31, 41] with the most notable feature that the charge density in the fluxes $H_{3} \wedge F_{6-p}$ goes to infinity (albeit in an integrable way). The sign of this infinity violates (2.4) and confirms the intuitive picture; the flux cloud is attracted both magnetically and gravitationally towards the source. Recently it was found [42] that this solution does preserve supersymmetry when $p=6$ (for other values of $p$ this is not yet investigated). This is a very interesting phenomenon and has implications for smearing and gauged supergravity [43]: there is no seven-dimensional gauged supergravity description of the $\mathrm{AdS}_{7}$ solution, regardless of whether the D6 branes are smeared or localised. Similarly, one can demonstrate that the smeared solution is not supersymmetric [43].

\section{Domain walls in the smeared limit}

In this section we give the seven-dimensional supergravity, the consistent truncation we use, and we discuss domain wall solutions of this seven-dimensional theory.

We want to stress that solutions to the seven-dimensional gauged supergravity correspond to ten-dimensional solutions with sources that are smeared in the compact directions. This is because after the dimensional reduction to a gauged supergravity we make a consistent truncation to only the lowest mass modes. An orientifold plane source in ten dimensions that is localised in the internal dimensions has a delta-function term in the equations or motion, which can no longer hold if we keep only the lightest KK modes. For instance for the Bianchi identity of the Ramond two-form $F_{2}$ we have:

$$
\mathrm{d} F_{2}=F_{0} H_{3}+\rho_{6}(\vec{y}),
$$

where the charge distribution $\rho_{6}(\vec{y})=Q_{6} \delta_{O 6}\left(\vec{y}-\vec{y}_{p}\right)$ for an O6 source that is localised on the internal manifold with coordinates $\vec{y}$ at position $\vec{y}_{p}$. By restricting to only lowest mass modes of the KK tower after dimensional reduction, we are effectively truncating the KK modes to lowest order. Expanding the $\delta_{O 6}$ in Fourier modes, this means that we are replacing $\delta_{O 6} \rightarrow 1$ and the source is 'smeared': it has a uniform charge distribution $\rho_{6}(\vec{y})=Q_{p}$. 


\subsection{Seven-dimensional gauged supergravity}

Consider massive IIA supergravity with $H$ flux on $\mathbb{T}^{3} / \mathbb{Z}_{2}$ and a space-filling O6 source that sits at the 8 fixed points of the $\mathbb{Z}_{2}$ involution:

$$
(a, b, c), \quad \text { where } \quad a, b, c \in\{0,1 / 2\} .
$$

Dimensional reduction gives half-maximal seven-dimensional gauged supergravity without vector multiplets (vector multiplets can be added by adding D6-branes). This is a noscale supergravity whose vacuum breaks supersymmetry. Hence the effective field theory of the fluctuation around the vacuum is not a supergravity. The seven-dimensional gauged supergravity that is obtained from direct dimensional reduction is instead capturing a consistent subset of the ten-dimensional fields that are capable of describing the spontaneous supersymmetry breaking in the vacuum.

For the purpose of constructing solutions we only use a truncation of this theory to the seven-dimensional metric and two scalar fields, which was described in [37]. For the full dimensional reduction of the bosonic sector, we refer to [43].

The Ansatz for the consistent reduction is given by

$$
\begin{aligned}
\mathrm{d} s_{10}^{2} & =\mathrm{e}^{2 \alpha \varphi} \mathrm{d} s_{7}^{2}+\mathrm{e}^{2 \beta \varphi} \delta_{a b} \mathrm{~d} y^{a} \mathrm{~d} y^{b}, \\
H_{3} & =h \mathrm{~d} y^{1} \wedge \mathrm{d} y^{2} \wedge \mathrm{d} y^{3}, \\
F_{0} & =m,
\end{aligned}
$$

with

$$
\alpha=\frac{1}{4} \sqrt{\frac{3}{5}}, \quad \beta=-5 \alpha / 3,
$$

and $h$ is the H-flux quantum. We denote the dilaton as $\phi$. Tadpole cancellation requires us to take the tension of the O6-plane to be ${ }^{4}$

$$
T=-2 h m
$$

The only contribution to the scalar potential in seven dimensions comes from the Romans mass term $m$, the amount of $H$-flux denoted $h$ and the orientifold tension, combining into [43]

$$
V(\phi, \varphi)=\frac{1}{2}\left(h \mathrm{e}^{-\frac{1}{2} \phi+6 \alpha \varphi}-m \mathrm{e}^{\frac{5}{4} \phi+\alpha \varphi}\right)^{2} .
$$

The action describing this two-scalar truncation of half-maximal seven-dimensional gauged supergravity is

$$
S=\int\left(\star \mathcal{R}-\frac{1}{2} \star \mathrm{d} \phi \wedge \mathrm{d} \phi-\frac{1}{2} \star \mathrm{d} \varphi \wedge \mathrm{d} \varphi-\star V(\phi, \varphi)\right) .
$$

One can demonstrate that the other scalars in the theory decouple; they are free fields that do not enter the scalar potential (see [43]). Since these free scalar fields do not enter the superpotential for supersymmetric solutions, they are constant. ${ }^{5}$ Hence our truncation is not a restriction and we can classify all BPS solutions.

\footnotetext{
${ }^{4}$ Note that $h m$ is positive.

${ }^{5}$ In fact, this also holds for the fake supersymmetric domain walls defined in section 3.2.
} 


\subsection{BPS and fake BPS domain walls}

Let us first recall the concept of fake supersymmetry for domain wall solutions [44]. Consider a general action of $D$-dimensional gravity coupled to scalars of the form

$$
S=\int\left(\star \mathcal{R}-\frac{1}{2} G_{i j}(\phi) \star \mathrm{d} \phi^{i} \wedge \mathrm{d} \phi^{j}-\star V(\phi)\right) .
$$

Domain wall solutions in $D$ dimensions have Poincaré invariance in $D-1$ dimensions and depend on the transversal direction only. They are described by a metric of the form

$$
\mathrm{d} s_{D}^{2}=f^{2}(z) \mathrm{d} z^{2}+g(z)^{2} \eta_{\mu \nu} \mathrm{d} x^{\mu} \mathrm{d} x^{\nu},
$$

where $\eta$ is the metric on Minkowski space. The scalar fields only depend on the $z$ coordinate. A certain domain wall solution, given by $\phi^{i}(z), f(z), g(z)$ is called fake supersymmetric if there exists a function $W(\phi)$ on the scalar target space such that the solution obeys

$$
\begin{aligned}
\dot{\phi}^{i} & =-f G^{i j} \partial_{j} W, \\
\frac{\dot{g}}{g} & =\frac{1}{2(D-2)} f W .
\end{aligned}
$$

The domain wall solution is given by a first-order gradient flow set by the fake superpotential $W$. Consistency with the second-order equations of motion implies a relation between the fake superpotential $W$ and the potential $V$ :

$$
V(\phi)=\frac{1}{2} G^{i j} \partial_{i} W \partial_{j} W-\frac{D-1}{4(D-2)} W^{2} .
$$

Genuine supersymmetric solutions obey the above constraint for a very specific function $W$ which is the 'true' superpotential that appears in the supersymmetry transformations of the supergravity theory. Any other function $W$ that obeys (3.14) generates a solution of the second-order equations of motion through first-order flow equations. One can prove that not all solutions to the second-order equations of motion are fake supersymmetric, although a set of local first-order Hamilton-Jacobi equations always exist. Recently, it has been clarified when solutions are fake supersymmetric or not [45].

Now we go back to our model in $D=7$, with action (3.9). To write down the solutions it is convenient to consider the following base rotation to new scalars $x$ and $u$ [37]:

$$
\begin{aligned}
& \phi=-\frac{\sqrt{15}}{8} x+\frac{7}{8} u, \\
& \varphi=-\frac{7}{8} x-\frac{\sqrt{15}}{8} u .
\end{aligned}
$$

One can then construct a one-dimensional family of superpotentials as follows

$$
W(x, u)=\exp \left(-\sqrt{\frac{3}{5}} x\right)[|h| \exp (-u)+|m| \exp (+u)-c],
$$

with $c$ a constant. The true superpotential giving supersymmetric solutions has $c=0$ [37]. All other values for $c$ correspond to fake superpotentials; for $c \neq 0$ the corresponding domain wall flow is not supersymmetric. 
From here on we use the gauge

$$
f(z)=g^{-4}(z)
$$

A combination of the $\dot{x}$ equation with the $\dot{g}$ equation allows to solve $g$ in terms of $x$

$$
e^{x}=g^{2 \sqrt{15}} .
$$

The remaining $\dot{x}$ and $\dot{u}$ equations can be simplified with the Ansatz,

$$
e^{x}=\left(h_{1} h_{2}\right)^{\frac{1}{2} \sqrt{\frac{3}{5}}}, \quad e^{u}=\left(h_{1} / h_{2}\right)^{1 / 2},
$$

where $h_{1}$ and $h_{2}$ obey the following first order equations:

$$
\begin{aligned}
& \dot{h}_{1}+c \sqrt{h_{1} / h_{2}}=2|h|, \\
& \dot{h}_{2}+c \sqrt{h_{2} / h_{1}}=2|m| .
\end{aligned}
$$

When $c=0$ we find the supersymmetric solution

$$
h_{1}=2|h| z+\ell_{1}^{2}, \quad h_{2}=2|m| z+\ell_{2}^{2},
$$

where $\ell_{1}^{2}, \ell_{2}^{2}$ are positive integration constants. These solutions were constructed in [46]. For the restricted set of solutions with $|m| \ell_{1}^{2}=|h| \ell_{2}^{2}$, the warped extension of this solution was found in our previous paper [37]. In the following section we discuss the warped extension of generic BPS solutions with $|m| \ell_{1}^{2} \neq|h| \ell_{2}^{2}$.

We have not been able to solve analytically for the general fake supersymmetric solutions ${ }^{6}$ but it is possible to find numerical solutions. There is however a simple class of solutions that can be found explicitly for non-zero $c$. These solutions have constant scalar $u$ with $\exp (2 u)=\frac{|h|}{|m|}$ and only the scalar $x$ is flowing. They are determined by

$$
h_{1}(z)=\left(2|h|-c \sqrt{\frac{|h|}{|m|}}\right) z+\ell^{2}, \quad h_{2}(z)=\frac{|m|}{|h|} h_{1}(z) .
$$

Such solutions correspond to a flow through the minimum of the no-scale potential where the on-shell value of the scalar potential vanishes. When we tune the velocity of the scalar $x$ such that $c=0$, this is the BPS flow (3.22) with $|m| \ell_{1}^{2}=|h| \ell_{2}^{2}$. For other values of $c$ it is fake supersymmetric.

\section{$4 \quad$ Full backreaction in ten-dimensional supergravity}

In this section we construct the warped counterpart of the domain wall solutions of the previous section. The domain walls preserve half of the supersymmetry of the half-maximal gauged supergravity. Hence one is naturally lead to investigate 1/4 BPS solutions in massive IIA supergravity that have the proper symmetries to correspond to a domain wall

\footnotetext{
${ }^{6}$ By that we mean an expressions in terms of well known and simple functions.
} 
on the worldvolume of the O6-plane. Since there is a map between compact O6 solutions and non-compact D6 solutions as explained in section 2, we can rely on the extensive literature of BPS brane solutions in 10D supergravity. It turns out that the analysis performed in [39] is the key to understand the backreaction. We start by reviewing the quarter BPS solution of [39] in massive IIA supergravity in subsection 4.1. In subsection 4.2 we explicitly smear these BPS solutions and compare them to the uplift of the BPS domain walls in $7 \mathrm{D}$ gauged supergravity. This allows us to uniquely define their backreaction in subsection 4.3 .

\subsection{1/4 BPS solutions in massive IIA supergravity}

Interestingly a general analysis of the BPS equations for an Ansatz that describes domain walls inside the D6 worlvolume in massive IIA has been carried out earlier by Imamura in [39], where an expression for the general solution was shown to be of the following elegant form in string frame:

$$
\begin{aligned}
\mathrm{d} s^{2} & =S^{-1 / 2} \eta_{i j} \mathrm{~d} x^{i} \mathrm{~d} x^{j}+K S^{-1 / 2} \mathrm{~d} \tilde{z}^{2}+K S^{1 / 2} \mathrm{~d} s_{3}^{2}, \\
\mathrm{e}^{\phi} & =g_{s} K^{1 / 2} S^{-3 / 4}, \\
F_{2} & =-\frac{1}{g_{s}} \star_{3} \mathrm{~d}_{3} S, \\
H_{3} & =\frac{\partial}{\partial \tilde{z}}(K S) \star_{3} 1-\mathrm{d} \tilde{z} \wedge \star_{3} \mathrm{~d}_{3} K, \\
F_{0} & =m,
\end{aligned}
$$

where $\mathrm{d} s_{3}^{2}$ is the metric on $\mathbb{R}^{3} ; \star_{3}$ and $\mathrm{d}_{3}$ are the corresponding hodge star and differential. In all ten-dimensional solutions, we follow the conventions and equations of motion of massive IIA supergravity as in [31]. We have used the coordinate $\tilde{z}$, because it is not necessarily the previous domain wall coordinate $z$ introduced in (3.11). The relation will be established below. The Ansatz is given in terms of two functions $S(r, \tilde{z})$ and $K(r, \tilde{z})$ that are determined by the differential equations

$$
\begin{aligned}
\nabla_{3}^{2} S+\frac{1}{2} \frac{\partial^{2} S^{2}}{\partial \tilde{z}^{2}} & =-g_{s} Q_{6} \delta \\
m g_{s} K & =\frac{\partial S}{\partial \tilde{z}}
\end{aligned}
$$

with $\delta$ describing the localised source on $\mathbb{R}^{3}$. Note that this Ansatz is written formally as a D6-NS5 solution. The function $S$ appears with the correct factors for a D6 harmonic function, $K$ for an NS5 harmonic function.

Non-compact D-brane solutions. Let us consider two known solutions to the noncompact equations. The first is the ordinary D6 brane, which has only $r$-dependence

$$
S=1+\frac{g_{s} Q_{6}}{r} .
$$

As is well known, this solution preserves 16 supercharges rather than 8 . 
A second class of solutions has a linear $\tilde{z}$-dependence in $S$. This class is parameterised by two constants $g_{s}$ and $\alpha$, where $\alpha$ is the coefficient of the linear piece

$$
S=1+\frac{g_{s} Q_{6}}{r}-\frac{\alpha^{2} r^{2}}{6}+\alpha \tilde{z}, \quad K=\frac{\alpha}{m g_{s}} .
$$

This is the BPS solution of [38] where the following parameters were chosen: $g_{s}=1$, $\alpha=m$.

Compact orientifold solutions. We obtain the compact $O 6$ solution from the above non-compact solution by taking the transverse space to be $\mathbb{T}^{3} / \mathbb{Z}_{2}$. The compact solution has the usual caveat that the Laplacian-type equation as (4.2) on a compact space may not be explicitly solvable.

For the reader worried about this quick jump from non-compact to compact solutions we now prove that the tadpole condition is obeyed. Consider the part of the $H_{3}$ field that sits inside the transversal space:

$$
H_{\text {internal }}=\frac{1}{m g_{s}} \frac{1}{2} \frac{\partial^{2}}{\partial \tilde{z}^{2}} S^{2} \star_{3} 1 .
$$

We used the definition of $K$ (4.3) to rewrite the first term for the expression of $H$ in (4.1). Now we rewrite this once more using (4.2) such that we end up with

$$
H_{\text {internal }}=\frac{1}{m g_{s}}\left(-g_{s} Q_{6} \delta-\nabla_{3}^{2} S\right) \star_{1} .
$$

When we integrate $\mathrm{m} \mathrm{H}_{3}$ over the internal space, we have

$$
\int_{\mathbb{T}^{3} / \mathbb{Z}_{2}} m H_{\text {internal }}=-Q_{6}-\frac{1}{g_{s}} \int_{\mathbb{T}^{3} / \mathbb{Z}_{2}} \nabla^{2} S .
$$

On a compact space the last term must be zero. Hence we automatically satisfy the tadpole condition (2.2) with Romans mass $F_{0}=m$.

\subsection{Smeared limit in 10 dimensions}

The smeared limit ('unwarped' limit) we perform is a uniform smearing over the 3-dimensional space. On the level of the ten-dimensional equations of motion, one starts by replacing the delta-functions in the source terms by 1 . This affects the Einstein equations, dilaton equation and the $F_{2}$ Bianchi identity. Solutions to these equations will now be such that nothing depends on the internal coordinates. Finally one replaces the O6 charge that multiplies the delta function by $Q_{6}=-h m$ (charge dissolved in flux), through the tadpole condition. One can easily show that the BPS equations (4.2) and (4.3) solve these equations if also in the BPS equation (4.2) the delta function is replaced by 1 :

$$
\frac{1}{2} \frac{\partial^{2} S^{2}}{\partial \tilde{z}^{2}}=g_{s} h m, \quad m g_{s} K=\frac{\partial S}{\partial \tilde{z}},
$$

where the $H_{3}$-flux is written as $H_{3}=h \mathrm{vol}_{3}$. As explained in the beginning of section 3 , the ten-dimensional uplift of solutions to seven-dimensional supergravity should correspond to such a smeared solution. 
We now verify that indeed the general $1 / 4$ BPS solution to the ten-dimensional equations of motion after smearing coincides with the uplift of the BPS solutions of sevendimensional supergravity determined by (3.22). The latter are given by

$$
\begin{aligned}
\mathrm{d} s^{2} & =h_{2}^{-\frac{1}{4}} \eta_{i j} \mathrm{~d} x^{i} \mathrm{~d} x^{j}+h_{1}^{-\frac{1}{2}} h_{2}^{-\frac{3}{4}} \mathrm{~d} \tilde{z}^{2}+h_{1}^{\frac{1}{2}} h_{2}^{-\frac{1}{4}} \delta_{a b} \mathrm{~d} y^{a} \mathrm{~d} y^{b}, \\
\mathrm{e}^{\phi} & =h_{1}^{\frac{1}{4}} h_{2}^{-\frac{5}{8}} \\
H_{3} & =h \mathrm{~d} y^{1} \wedge \mathrm{d} y^{2} \wedge \mathrm{d} y^{3},
\end{aligned}
$$

and $F_{2}=0$. Again we have chosen the gauge (3.17). Comparing to the Ansatz (4.1), we identify:

$$
S=g_{s} h_{2}^{\frac{1}{2}}, \quad K=g_{s}^{-1 / 2} h_{1}^{\frac{1}{2}} h_{2}^{-\frac{1}{2}},
$$

and we have the following relation between the coordinates

$$
h_{1}^{-1 / 2} \mathrm{~d} z=g_{s}^{-1 / 2} \mathrm{~d} \tilde{z},
$$

which can be integrated to $\tilde{z}=g_{s}^{1 / 2}|h|^{-1} \sqrt{2|h| z+\ell_{1}^{2}}$. One then readily verifies that $S$ and $K$ solve equations (4.9). This establishes the consistency of our smearing procedure.

\subsection{Warped domain walls}

We now discuss the solution with O6 sources localised on the three-dimensional space.

\subsubsection{Warping for a simple class of domain walls}

One explicit backreacted solution is known and is given by equation (4.5). This solution is to be interpreted as a solution for which the scalar fields flow through the moduli space of the no-scale Minkowski vacuum [37]. This means that the corresponding domain wall solutions in the seven-dimensional gauged supergravity are the ones that have $m \ell_{1}^{2}=h \ell_{2}^{2}$, since those solutions have $V=0$ throughout their flow (see discussion around (3.23)).

The linear $\tilde{z}$ dependence is not altered by warping and this is a simple consequence of the warped effective potential and the gauged supergravity potential both keeping the same minimal value for the on-shell scalar flow [47]. The rough behaviour is shown in figure 1 . This viewpoint furthermore explains why ordinary D6 branes, without Romans mass and $H_{3}$ flux, allow a (non-supersymmetric) extension for which a piece is added to the harmonic that is linear in the worldvolume coordinates (the so-called dynamical branes $[48,49]$ )

$$
H(r)=1+\frac{Q_{6}}{r}+\sum_{i=0}^{6} c_{i} x^{i}
$$

When interpreted as a 'compactification' without flux, there is no scalar potential. The linear dependence comes in through the profile of a free scalar on the worldvolume. It is the same as for solutions with flux that have a flow is in the moduli space of the vacuum. 


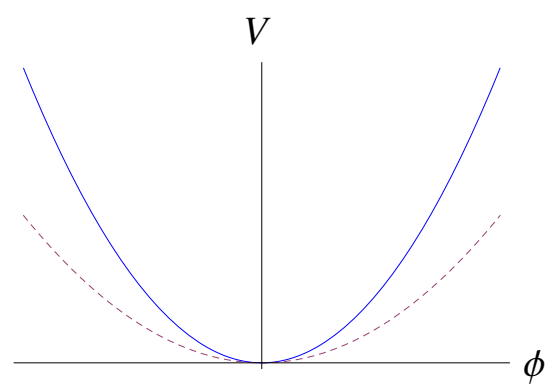

Figure 1. The scalar potential in the gauged supergravity is depicted with the full line. The dashed line represents the potential in the warped effective field theory.

\subsubsection{Warping for all supersymmetric domain walls}

Using the map of orientifold solutions to D-branes, we treat the non-compact D-brane solution first. We consider a D6-brane source at the origin of the three-dimensional transverse space, such that the solution only depends on the three-dimensional radius $r$ and of course also on the domain wall coordinate $\tilde{z}$. A useful suggestion for tackling this problem has been made in [39]. We write the solution as a Laurent series with leading $1 / r$ singular piece

$$
S(r, \tilde{z})=\sum_{n=-1}^{\infty} a_{n}(\tilde{z}) r^{n} .
$$

The rationale for this specific Laurent series sum is that the $1 / r$ contribution gives the deltafunction pole in the $S$-equation; the higher order terms cannot be excluded a priori. We do not expect any higher order $1 / r^{k}$ poles. This Ansatz, when plugged into the equations of motion, gives a recursive relation for the $\tilde{z}$ dependent coefficients [39]

$$
n(n+1) a_{n}=-\frac{1}{2} \frac{\partial^{2}}{\partial \tilde{z}^{2}} \sum_{k=0}^{n} a_{k-1} a_{n-k-1} .
$$

We can solve this series by fixing the first two terms $a_{-1}, a_{0}$. All the higher order terms are determined recursively. The sum (4.15) for $n=0$ gives $\partial_{\tilde{z}}^{2} a_{-1}=0$ and leaves $a_{0}$ free. We fix these through the appropriate limits. The first term is fixed by the delta-function pole to be $\tilde{z}$ independent since it has to equal the O6/D6 charge:

$$
a_{-1}=g_{s} Q_{6} .
$$

The second term can be constrained by noting that it must be such that upon smearing, the $H_{3}$ flux becomes $\tilde{z}$ independent and is given by the flux quantum we dubbed $h$ before: $H_{3}=h \star_{3}$ 1. This implies $S(r, \tilde{z}) \rightarrow S(\tilde{z})=a_{0}(\tilde{z})$ and

$$
\frac{1}{2} \frac{\partial^{2}}{\partial \tilde{z}^{2}} a_{0}^{2}=g_{s} m h
$$

By shifting the origin of the $\tilde{z}$-axis, the most general solution is

$$
a_{0}=\sqrt{g_{s} m h \tilde{z}^{2}+\beta},
$$


with one integration constant $\beta$. By comparison to the smeared limit (4.12), we see that the integration constant is

$$
\beta=\frac{g_{s}^{2}}{h}\left(h \ell_{2}^{2}-m \ell_{1}^{2}\right)
$$

and it denotes the "distance" from the backreacted solutions with $m \ell_{1}=h \ell_{2}$ we discussed in [37], see eq. (4.5).

For the backreaction with most general $r$-dependence, we need the other terms $a_{n}$. They are fixed by recursion and this uniquely defines the backreaction of the domain wall solutions of seven-dimensional supergravity. The first terms are:

$$
\begin{aligned}
& a_{1}=-\beta \frac{g_{s} m h Q_{6}}{2 a_{0}^{3}}, \\
& a_{2}=-\frac{1}{6} g_{s} m h+\beta\left(g_{s}^{2} m h Q_{6}\right)^{2}\left(\frac{1}{a_{0}^{5}}-\frac{5 \beta}{4 a_{0}^{7}}\right), \\
& a_{3}=\beta\left(g_{s} m h\right)^{2}\left(g_{s} Q_{6}\right)\left(\frac{1}{4 a_{0}^{4}}-\frac{\beta}{3 a_{0}^{6}}\right)+\beta\left(g_{s}^{2} m h Q_{6}\right)^{3}\left(-\frac{5}{2 a_{0}^{7}}+\frac{35 \beta}{4 a_{0}^{9}}-\frac{105 \beta^{2}}{16 a_{0}^{11}}\right) .
\end{aligned}
$$

When $\beta=0$, the $a_{n}$ series terminates at order 2 , the only non-trivial extra term is $a_{2}=$ $-g_{s} m h / 6$. Then we find the backreacted solution with $m \ell_{2}^{2}=h \ell_{1}^{2}$ described in (4.5). However, for the most general solution with $\beta \neq 0$ or equivalently $m \ell_{2}^{2}=h \ell_{1}^{2}$, the series is infinite.

For the compact solution on $T^{3} / \mathbb{Z}_{2}$ with orientifold sources, the Laurent expansion should be replaced by a Fourier expansion

$$
S=\sum_{\vec{n}} a_{\vec{n}}(\tilde{z}) \exp [i \vec{n} \cdot \vec{\theta}]
$$

where $\vec{\theta}$ are the angles of the 3-torus. Assuming reality of the function $S$ and proper parity under the orientifold involution restricts the choice of $\tilde{z}$-dependent Fourier coefficients $a_{n}$, but we were unable to find a simple iterative equation for them. This is an interesting unsolved problem, which, if solvable, would lead to explicit solutions for KK modes.

\section{$5 \quad$ A $4 \mathrm{D}$ example from $\mathrm{O} 3$ planes}

The seven-dimensional solutions studied above are mostly a toy model for the situation of genuine interest: flux compactifications to $D=4$. However, by simply T-dualising the seven-dimensional model we obtain a four-dimensional model.

The T-dualised model describes an O3 compactification of IIB supergravity on a $\mathbb{T}^{6}$ (or an orbifold thereof) to four dimensions. The three-form fluxes take a simple form. We fill one three-torus inside $\mathbb{T}^{6}$ with $H$-flux and the orthogonal three-torus with $F_{3}$ flux. This is perhaps the simplest compactification of the kind discussed in [8]. Fluxes of this kind necessary lead to a non-BPS Minkowski vacuum [37]. The BPS domain wall solutions of the $4 \mathrm{D}$ supergravity were constructed in [37] and we briefly recall those solutions before discussing the warped extension that arises when the $\mathrm{O} 3$ plane is localised. 


\subsection{Smeared O3 compactification}

The discussion parallels that of section 3. However, now we need to consider a truncation of the compactification to three scalars: the dilaton $\phi$, the volume modulus $v$ of the internal six-torus and the volume modulus of the 3 -torus threaded by the $F_{3}$-flux, called $\chi$. The volume of the orthogonal three-torus, filled with $H_{3}$ depends on $v$ and $\chi$. The Ansatz, in 10-dimensional Einstein frame, is given by

$$
\begin{aligned}
\mathrm{d} s_{10}^{2} & =\exp (2 \alpha v) \mathrm{d} s_{4}^{2}+\exp (2 \beta v)\left\{\exp (\gamma \chi) \delta_{a b} \mathrm{~d} y^{a} \mathrm{~d} y^{b}+\exp (-\gamma \chi) \delta_{i j} \mathrm{~d} y^{i} \mathrm{~d} y^{j}\right\} \\
H_{3} & =h \mathrm{~d} y^{1} \wedge \mathrm{d} y^{2} \wedge \mathrm{d} y^{3} \\
F_{3} & =m \mathrm{~d} y^{4} \wedge \mathrm{d} y^{5} \wedge \mathrm{d} y^{6}
\end{aligned}
$$

We used the following notation: $m$ and $h$ are flux quanta and they are constrained by the tadpole condition as $T_{O 3}=-h m$, the indices $a, b$ run from 1 to 3 and $i, j$ from 4 to 6 . The numbers $\alpha, \beta, \gamma$ are chosen such that we end up in lower-dimensional Einstein frame with canonically normalised fields:

$$
\beta=-\frac{1}{3} \alpha, \quad \alpha^{2}=\frac{3}{16}, \quad \gamma^{2}=\frac{1}{3} .
$$

The lower-dimensional action is obtained from a direct dimensional reduction

$$
S=\int \sqrt{-g}\left(R-\frac{1}{2}(\partial \phi)^{2}-\frac{1}{2}(\partial \chi)^{2}-\frac{1}{2}(\partial v)^{2}-V(\phi, v, \chi)\right) .
$$

The scalar potential $V$ gets contributions from the fluxes and the negative orientifold plane tension and has the form of an exact square (due to the tadpole condition). To write it down in a clean way we perform the following $\mathrm{SO}(3)$ field rotation $(\phi, v, \chi) \rightarrow(x, u, w)$ :

$$
\begin{aligned}
& \phi=\frac{1}{2} u+\frac{\sqrt{3}}{2} w, \\
& v=-x, \\
& \chi=-\frac{\sqrt{3}}{2} u+\frac{1}{2} w .
\end{aligned}
$$

The scalar potential then becomes [37]

$$
V(x, u, w)=\frac{1}{2} \exp (-\sqrt{3} x)[|h| \exp (-u)-|m| \exp (+u)]^{2},
$$

The scalar potential only depends on two scalars $\left(u\right.$ and $x$ ). T-duality along $y^{4}, y^{5}, y^{6}$ reproduces the $\mathrm{O} 6$ compactification of massive IIA.

\subsection{BPS domain walls from smeared O3 planes}

The real superpotential that determines the BPS domain wall solutions is given by

$$
W=\exp (-\sqrt{3} x / 2)[|h| \exp (-u)+|m| \exp (+u)] .
$$

We present the solutions in the following metric gauge

$$
\mathrm{d} s_{4}^{2}=g^{-2}(z) \mathrm{d} z^{2}+g(z)^{2} \eta_{\mu \nu} \mathrm{d} x^{\mu} \mathrm{d} x^{\nu},
$$


where $\eta$ is the metric on $3 \mathrm{~d}$ Minkowski space. We find [37]:

$$
\begin{aligned}
g & =\left(h_{1} h_{2}\right)^{\frac{1}{8}}, \\
\exp (x) & =\left(h_{1} h_{2}\right)^{\sqrt{3} / 4}, \\
\exp (u) & =\left(\frac{h_{1}}{h_{2}}\right)^{1 / 2} .
\end{aligned}
$$

where, as before,

$$
h_{1}=2|h| z+\ell_{1}^{2}, \quad h_{2}=2|m| z+\ell_{2}^{2} .
$$

\subsection{Warped solutions from localised O3 planes}

By T-dualising the 1/4 BPS solutions with lcoalised D6/O6 sources we obtain warped solutions in four-dimensions with D3/O3 sources that are localised in three directions, but smeared over the three T-duality directions $y^{4}, y^{5}, y^{6}$ :

$$
\begin{aligned}
\mathrm{d} s^{2} & =S^{-1 / 2} \eta_{\mu \nu} \mathrm{d} x^{\mu} \mathrm{d} x^{\nu}+K S^{-1 / 2} \mathrm{~d} \tilde{z}^{2}+K S^{1 / 2} \mathrm{~d} s_{3}^{2}+S^{1 / 2} \delta_{i j} \mathrm{~d} y^{i} \mathrm{~d} y^{j}, \\
\mathrm{e}^{\phi} & =g_{s} K^{1 / 2}, \\
F_{5} & =-\frac{1}{g_{s}} \star_{3} \mathrm{~d}_{3} S \wedge \mathrm{d} y_{4} \wedge \mathrm{d} y_{5} \wedge \mathrm{d} y_{6}, \\
H_{3} & =\frac{\partial}{\partial \tilde{z}}(K S) \star_{3} 1-\mathrm{d} \tilde{z} \wedge \star_{3} \mathrm{~d}_{3} K, \\
F_{3} & =m \mathrm{~d} y_{4} \wedge \mathrm{d} y_{5} \wedge \mathrm{d} y_{6} .
\end{aligned}
$$

Note that the dilaton does not depend on $S$.

We expect the domain wall solutions with general ISD fluxes to be much more involved. A more formal method, as in [50], might be useful for finding them.

\section{Discussion}

In this paper we explored domain wall solutions in no-scale gauged supergravities that have an interpretation in terms of orientifold compactifications of type II supergravity. The gauged supergravity does not account for the full backreaction of the orientifold planes. Accordingly, the domain wall solutions solve the ten-dimensional equations of motion only when the orientifolds are smeared over the extra dimensions. We have written down the Ansatz and the first-order BPS equations for the domain wall solutions in 10-dimensional supergtravity with localised sources. In order to find explicit expressions we rely on a formal map between compact orientifold solutions and non-compact D brane solutions. This allows us to benefit from existing results on BPS brane solutions with fluxes, such as those in $[38,39]$. Our analysis gives an interpretation to part of the BPS brane solutions of $[38,39]$ as describing domain walls inside the worldvolume of D-branes. In order to be as explicit as possible, we first considered a very simple no-scale orientifold compactification: the O6 reduction of massive IIA to $D=7[12,37]$. In section 5 we have demonstrated how a formal T-duality relates this to the more interesting IIB toroidal orientifold compactifications to 
$D=4$. The general IIB toroidal orientifold would be interesting to work out in detail. There are many other directions that this work naturally leads to and we list them here.

It would be interesting to find non-supersymmetric solutions and whether backreaction can even be computed in this case. We have reasons to believe that for fake BPS solutions this should be possible. In fact one example already exist and was presented in [38]. These are simple supersymmetry-breaking domain walls that flow through moduli space and it is possible to match them to fake BPS domain walls of the gauged supergravity.

The research avenues we address here fit into a bigger question: what are the backreacted versions of all gauged supergravity solutions? We can think of cosmological solutions, black holes and so on. In practice this means understanding the zoo of configurations inside the worldvolume of branes from a 10-dimensional point of view.

One could also wonder whether our computations could help in understanding warped effective field theory. The difficulty of formulating WEFT is performing the integration of the action over the extra dimensions and trying to identify the lower-dimensional degrees of freedom. With our approach we have the explicit 10D BPS equations and perhaps the integrated versions of these equations give the BPS equations of the WEFT, leading to the action.

\section{Acknowledgments}

We would like to thank Iosif Bena, Gary Shiu and Timm Wrase for useful discussions. TVR is supported by a Pegasus fellowship and by the Odysseus programme of the FWO. The work of JB is supported by the Swedish Research Council (VR), and the Göran Gustafsson Found. The work of BJ was partially supported by the M.E.C. under contract FIS2010-17395 and by the Junta de Andalucía groups P07-FQM 03048 and FQM-6552. BV acknowledges support from the European Union through the Marie Curie Intra-European fellowship 328652-QM-sing and would like to thank Evelien Dejonghe. For the initial work on this project, BV was supported by the ERC Starting Independent Researcher Grant 240210-String-QCD-BH.

\section{A $\mathcal{N}=1$ supergravity formulation}

In what follows we derive the real superpotential in four dimensions from the standard $\mathcal{N}=1$ supergravity formulation for IIB orientifold compactfications with 3 -form fluxes [8]. Such a formulation gives a complex superpotential $\mathcal{W}$ and a real Kähler potential $\mathcal{K}$. The usual convention found in the literature is

$$
S=\int \frac{1}{2} \star R-\mathcal{K}_{i \bar{j}} \star \mathrm{d} \Phi^{i} \wedge \mathrm{d} \bar{\Phi}^{j}-\star V
$$

where

$$
\begin{aligned}
\mathcal{K}_{i \bar{j}} & =\partial_{i} \partial_{\bar{j}} \mathcal{K}, \\
D_{i} \mathcal{W} & =\partial_{i} \mathcal{W}+\mathcal{W} \partial_{i} \mathcal{K} .
\end{aligned} \quad V=\mathrm{e}^{\mathcal{K}}\left(D_{i} \mathcal{W} \overline{D_{j} \mathcal{W}} \mathcal{K}^{i \bar{j}}-3|W|^{2}\right),
$$


In a real formulation for the scalars, with real field space metric $G_{i j}$, we find that the function $T=\left|e^{\mathcal{K} / 2} \mathcal{W}\right|$ obeys

$$
V=G^{i j} \partial_{i} T \partial_{j} T-3 T^{2}
$$

If we then perform the following rescalings (change of conventions) $V \rightarrow \frac{1}{2} V, G_{i j} \rightarrow \frac{1}{4} G_{i j}$ we indeed find that $T$ is the real superpotential $W$ in the conventions (3.10), (3.14) up to a factor: $T=\frac{1}{\sqrt{8}} W$.

The complex superpotential and the real Kähler potential are given by [8]

$$
\begin{aligned}
\mathcal{W} & =\int_{V_{6}} \Omega \wedge G, \\
\mathcal{K} & =-\ln [i(\tau-\bar{\tau})]-3 \ln [-i(\rho-\bar{\rho})],
\end{aligned}
$$

where the integral is over the six-dimensional internal manifold, $\Omega$ is the complex holomorphic 3-form and $G$ is the complex 3-form $G=F_{3}-\tau H_{3}$, where $\tau=C_{0}+i \mathrm{e}^{-\phi}$ and $\rho=b+i \mathrm{e}^{-4 \alpha v / 3}$. The axions $b$ and $C_{0}$ are put to zero. The geometry can be characterized by the following choice of Kähler form $J$ and holomorphic 3-form $\Omega=\Omega_{R}+i \Omega_{I}:^{7}$

$$
\begin{aligned}
\Omega_{R}= & \mathrm{e}^{-3 \beta v / 2}\left[\mathrm{e}^{-3 \gamma \chi / 2} \mathrm{~d} y^{4} \wedge \mathrm{d} y^{5} \wedge \mathrm{d} y^{6}+\mathrm{e}^{\gamma \chi / 2} \mathrm{~d} y^{2} \wedge \mathrm{d} y^{3} \wedge \mathrm{d} y^{6}+\right. \\
& \left.\mathrm{e}^{\gamma \chi / 2} \mathrm{~d} y^{1} \wedge \mathrm{d} y^{3} \wedge \mathrm{d} y^{4}+\mathrm{e}^{\gamma \chi / 2} \mathrm{~d} y^{1} \wedge \mathrm{d} y^{2} \wedge \mathrm{d} y^{5}\right] \\
\Omega_{I}= & \mathrm{e}^{-3 \beta v / 2}\left[\mathrm{e}^{3 \gamma \chi / 2} \mathrm{~d} y^{1} \wedge \mathrm{d} y^{2} \wedge \mathrm{d} y^{3}+\mathrm{e}^{-\gamma \chi / 2} \mathrm{~d} y^{1} \wedge \mathrm{d} y^{4} \wedge \mathrm{d} y^{5}-\right. \\
& \left.\left.\mathrm{e}^{-\gamma \chi / 2} \mathrm{~d} y^{2} \wedge \mathrm{d} y^{5} \wedge \mathrm{d} y^{6}-\mathrm{e}^{-\gamma \chi / 2} \mathrm{~d} y^{3} \wedge \mathrm{d} y^{4} \wedge \mathrm{d} y^{6}\right)\right] \\
J= & \mathrm{e}^{-\beta v}\left[\mathrm{~d} y^{1} \wedge \mathrm{d} y^{6}+\mathrm{d} y^{2} \wedge \mathrm{d} y^{4}+\mathrm{d} y^{3} \wedge \mathrm{d} y^{5}\right]
\end{aligned}
$$

This indeed reproduces the internal metric (5.1), as can be verified from $g_{m n}=-I_{m}^{l} J_{l n}$ where $I$ is the complex structure:

$$
I_{k}^{l}=c \varepsilon^{m_{1} m_{2} m_{3} m_{4} m_{5} l}\left(\Omega_{R}\right)_{k m_{1} m_{2}}\left(\Omega_{R}\right)_{m_{3} m_{4} m_{5}},
$$

with $c$ a constant, such that $I^{2}=-\mathbb{1}$. One now readily checks that $T$ coincides with the real superpotential $W$ of equation (5.10) up to an overall factor.

Open Access. This article is distributed under the terms of the Creative Commons Attribution License (CC-BY 4.0), which permits any use, distribution and reproduction in any medium, provided the original author(s) and source are credited.

\section{References}

[1] M. Petrini, G. Solard and T. Van Riet, AdS vacua with scale separation from IIB supergravity, JHEP 11 (2013) 010 [arXiv:1308.1265] [INSPIRE].

[2] D. Tsimpis, Supersymmetric AdS vacua and separation of scales, JHEP 08 (2012) 142 [arXiv: 1206.5900] [INSPIRE].

[3] B. de Wit, D.J. Smit and N.D. Hari Dass, Residual Supersymmetry of Compactified D $=10$ Supergravity, Nucl. Phys. B 283 (1987) 165 [InSPIRE].

\footnotetext{
${ }^{7}$ We use the following conventions: $\Omega \wedge \bar{\Omega}=\frac{4 i}{3} J \wedge J \wedge J=8 i$ vol $_{6}$.
} 
[4] J.M. Maldacena and C. Núñez, Supergravity description of field theories on curved manifolds and a no go theorem, Int. J. Mod. Phys. A 16 (2001) 822 [hep-th/0007018] [InSPIRE].

[5] P.K. Townsend, Cosmic acceleration and M-theory, hep-th/0308149 [INSPIRE].

[6] T. Wrase and M. Zagermann, On Classical de Sitter Vacua in String Theory, Fortsch. Phys. 58 (2010) 906 [arXiv: 1003.0029] [inSPIRE].

[7] U.H. Danielsson, S.S. Haque, G. Shiu and T. Van Riet, Towards Classical de Sitter Solutions in String Theory, JHEP 09 (2009) 114 [arXiv:0907.2041] [INSPIRE].

[8] S.B. Giddings, S. Kachru and J. Polchinski, Hierarchies from fluxes in string compactifications, Phys. Rev. D 66 (2002) 106006 [hep-th/0105097] [INSPIRE].

[9] S. Kachru, R. Kallosh, A.D. Linde and S.P. Trivedi, de Sitter vacua in string theory, Phys. Rev. D 68 (2003) 046005 [hep-th/0301240] [INSPIRE].

[10] R. Blumenhagen, M. Cvetič, P. Langacker and G. Shiu, Toward realistic intersecting D-brane models, Ann. Rev. Nucl. Part. Sci. 55 (2005) 71 [hep-th/0502005] [inSPIRE].

[11] R. Blumenhagen, B. Körs, D. Lüst and S. Stieberger, Four-dimensional String Compactifications with D-branes, Orientifolds and Fluxes, Phys. Rept. 445 (2007) 1 [hep-th/0610327] [INSPIRE].

[12] J. Blaback et al., Smeared versus localised sources in flux compactifications, JHEP 12 (2010) 043 [arXiv: 1009.1877] [INSPIRE].

[13] M. Graña, R. Minasian, M. Petrini and A. Tomasiello, A Scan for new $N=1$ vacua on twisted tori, JHEP 05 (2007) 031 [hep-th/0609124] [INSPIRE].

[14] O. DeWolfe, A. Giryavets, S. Kachru and W. Taylor, Type IIA moduli stabilization, JHEP 07 (2005) 066 [hep-th/0505160] [InSPIRE].

[15] C. Caviezel et al., The Effective theory of type IIA AdS $S_{4}$ compactifications on nilmanifolds and cosets, Class. Quant. Grav. 26 (2009) 025014 [arXiv:0806.3458] [INSPIRE].

[16] J.-P. Derendinger, C. Kounnas, P.M. Petropoulos and F. Zwirner, Superpotentials in IIA compactifications with general fluxes, Nucl. Phys. B 715 (2005) 211 [CERN-PH-TH-2004-228] [hep-th/0411276].

[17] K. Behrndt and M. Cvetič, General $N=1$ supersymmetric flux vacua of (massive) type IIA string theory, Phys. Rev. Lett. 95 (2005) 021601 [hep-th/0403049] [INSPIRE].

[18] C. Caviezel, T. Wrase and M. Zagermann, Moduli Stabilization and Cosmology of Type IIB on SU(2)-Structure Orientifolds, JHEP 04 (2010) 011 [arXiv:0912.3287] [INSPIRE].

[19] G. Solard, $N=1$ SUSY AdS $S_{4}$ vacua in IIB SUGRA on group manifolds, JHEP 02 (2014) 017 [arXiv: 1310.4836] [INSPIRE].

[20] B.S. Acharya, F. Benini and R. Valandro, Fixing moduli in exact type IIA flux vacua, JHEP 02 (2007) 018 [hep-th/0607223] [INSPIRE].

[21] F. Saracco and A. Tomasiello, Localized O6-plane solutions with Romans mass, JHEP 07 (2012) 077 [arXiv: 1201.5378] [InSPIRE].

[22] J. McOrist and S. Sethi, M-theory and Type IIA Flux Compactifications, JHEP 12 (2012) 122 [arXiv: 1208.0261] [INSPIRE].

[23] T. Banks and K. van den Broek, Massive IIA flux compactifications and U-dualities, JHEP 03 (2007) 068 [hep-th/0611185] [INSPIRE]. 
[24] L. Martucci, On moduli and effective theory of $N=1$ warped flux compactifications, JHEP 05 (2009) 027 [arXiv: 0902 .4031] [INSPIRE].

[25] A.R. Frey and J. Roberts, The Dimensional Reduction and Kähler Metric of Forms In Flux and Warping, JHEP 10 (2013) 021 [arXiv:1308.0323] [INSPIRE].

[26] G. Shiu, G. Torroba, B. Underwood and M.R. Douglas, Dynamics of Warped Flux Compactifications, JHEP 06 (2008) 024 [arXiv:0803.3068] [INSPIRE].

[27] A.R. Frey, G. Torroba, B. Underwood and M.R. Douglas, The Universal Kähler Modulus in Warped Compactifications, JHEP 01 (2009) 036 [arXiv: 0810.5768] [INSPIRE].

[28] J. Blaback, U.H. Danielsson and T. Van Riet, Resolving anti-brane singularities through time-dependence, JHEP 02 (2013) 061 [arXiv:1202.1132] [INSPIRE].

[29] A. Ceresole, G. Dall'Agata, A. Giryavets, R. Kallosh and A.D. Linde, Domain walls, near-BPS bubbles and probabilities in the landscape, Phys. Rev. D 74 (2006) 086010 [hep-th/0605266] [INSPIRE].

[30] P. Ahlqvist et al., Conifolds and Tunneling in the String Landscape, JHEP 03 (2011) 119 [arXiv: 1011.6588] [INSPIRE].

[31] J. Blaback et al., The problematic backreaction of SUSY-breaking branes, JHEP 08 (2011) 105 [arXiv: 1105.4879] [INSPIRE].

[32] P. McGuirk, G. Shiu and Y. Sumitomo, Non-supersymmetric infrared perturbations to the warped deformed conifold, Nucl. Phys. B 842 (2011) 383 [arXiv:0910.4581] [InSPIRE].

[33] I. Bena, M. Graña and N. Halmagyi, On the Existence of Meta-stable Vacua in Klebanov-Strassler, JHEP 09 (2010) 087 [arXiv:0912.3519] [INSPIRE].

[34] I. Bena, M. Graña, S. Kuperstein and S. Massai, Anti-D3 Branes: Singular to the bitter end, Phys. Rev. D 87 (2013) 106010 [arXiv:1206.6369] [InSPIRE].

[35] F.F. Gautason, D. Junghans and M. Zagermann, Cosmological Constant, Near Brane Behavior and Singularities, JHEP 09 (2013) 123 [arXiv:1301.5647] [INSPIRE].

[36] D. Junghans, Backreaction of Localised Sources in String Compactifications, arXiv:1309.5990 [INSPIRE].

[37] J. Blaback, B. Janssen, T. Van Riet and B. Vercnocke, Fractional branes, warped compactifications and backreacted orientifold planes, JHEP 10 (2012) 139 [arXiv: 1207.0814] [INSPIRE].

[38] B. Janssen, P. Meessen and T. Ortín, The D8-brane tied up: String and brane solutions in massive type IIA supergravity, Phys. Lett. B 453 (1999) 229 [hep-th/9901078] [INSPIRE].

[39] Y. Imamura, 1/4 BPS solutions in massive IIA supergravity, Prog. Theor. Phys. 106 (2001) 653 [hep-th/0105263] [INSPIRE].

[40] I.R. Klebanov and M.J. Strassler, Supergravity and a confining gauge theory: Duality cascades and chi SB resolution of naked singularities, JHEP 08 (2000) 052 [hep-th/0007191] [INSPIRE].

[41] J. Blaback et al., (Anti-)Brane backreaction beyond perturbation theory, JHEP 02 (2012) 025 [arXiv: 1111.2605] [INSPIRE].

[42] F. Apruzzi, M. Fazzi, D. Rosa and A. Tomasiello, All AdS $S_{7}$ solutions of type-II supergravity, JHEP 04 (2014) 064 [arXiv: 1309.2949] [InSPIRE]. 
[43] U.H. Danielsson, G. Dibitetto, M. Fazzi and T. Van Riet, A note on smeared branes in flux vacua and gauged supergravity, JHEP 04 (2014) 025 [arXiv: 1311.6470] [INSPIRE].

[44] D.Z. Freedman, C. Núñez, M. Schnabl and K. Skenderis, Fake supergravity and domain wall stability, Phys. Rev. D 69 (2004) 104027 [hep-th/0312055] [INSPIRE].

[45] M. Trigiante, T. Van Riet and B. Vercnocke, Fake supersymmetry versus Hamilton-Jacobi, JHEP 05 (2012) 078 [arXiv: 1203.3194] [INSPIRE].

[46] E. Bergshoeff, M. Nielsen and D. Roest, The Domain walls of gauged maximal supergravities and their M-theory origin, JHEP 07 (2004) 006 [hep-th/0404100] [INSPIRE].

[47] M.R. Douglas, J. Shelton and G. Torroba, Warping and supersymmetry breaking, arXiv:0704.4001 [INSPIRE].

[48] P. Binetruy, M. Sasaki and K. Uzawa, Dynamical D4-D8 and D3-D7 branes in supergravity, Phys. Rev. D 80 (2009) 026001 [arXiv:0712.3615] [InSPIRE].

[49] G.W. Gibbons, H. Lü and C.N. Pope, Brane worlds in collision, Phys. Rev. Lett. 94 (2005) 131602 [hep-th/0501117] [INSPIRE].

[50] M. Haack, D. Lüst, L. Martucci and A. Tomasiello, Domain walls from ten dimensions, JHEP 10 (2009) 089 [arXiv:0905.1582] [INSPIRE]. 